\title{
Cemented, cementless, and hybrid prostheses for total hip replacement: cost effectiveness analysis
}

\author{
(c) $(1)(9)$ OPEN ACCESS
}

\author{
Mark Pennington lecturer in health economics ${ }^{1}$, Richard Grieve reader in health economics ${ }^{1}$, Jasjeet \\ S Sekhon professor ${ }^{2}$, Paul Gregg professor and consultant orthopaedic surgeon ${ }^{3}$ vice chairman ${ }^{4}$, \\ Nick Black professor of health services research ${ }^{1}$, Jan $\mathrm{H}$ van der Meulen professor of clinical \\ epidemiology ${ }^{1}$
}

${ }^{1}$ Department of Health Services Research and Policy, London School of Hygiene and Tropical Medicine, London WC1H 9SH, UK; ${ }^{2}$ Travers Department of Political Science, Department of Statistics, Center for Causal Inference and Program Evaluation, Institute of Governmental Studies, University of California, Berkeley, CA, USA; ${ }^{3}$ James Cook Hospital, South Tees Hospitals NHS Foundation Trust, Middlesbrough, UK; ${ }^{4}$ National Joint Registry for England and Wales, Healthcare Quality Improvement Partnership, London, UK

\begin{abstract}
Objective To compare the cost effectiveness of the three most commonly chosen types of prosthesis for total hip replacement.

Design Lifetime cost effectiveness model with parameters estimated from individual patient data obtained from three large national databases.

Setting English National Health Service.

Participants Adults aged 55 to 84 undergoing primary total hip replacement for osteoarthritis.

Interventions Total hip replacement using either cemented, cementless, or hybrid prostheses.

Main outcome measures Cost $(£)$, quality of life (EQ-5D-3L, where 0 represents death and 1 perfect health), quality adjusted life years (QALYs), incremental cost effectiveness ratios, and the probability that each prosthesis type is the most cost effective at alternative thresholds of willingness to pay for a QALY gain.
\end{abstract}

Results Lifetime costs were generally lowest with cemented prostheses, and postoperative quality of life and lifetime QALYs were highest with hybrid prostheses. For example, in women aged 70 mean costs were $£ 6900$ ( $\$ 11000$; €8200) for cemented prostheses, $£ 7800$ for cementless prostheses, and $£ 7500$ for hybrid prostheses; mean postoperative EQ-5D scores were $0.78,0.80$, and 0.81 , and the corresponding lifetime QALYs were 9.0, 9.2, and 9.3 years. The incremental cost per QALY for hybrid compared with cemented prostheses was $£ 2500$. If the threshold willingness to pay for a QALY gain exceeded $£ 10000$, the probability that hybrid prostheses were most cost effective was about $70 \%$. Hybrid prostheses have the highest probability of being the most cost effective in all subgroups, except in women aged 80 , where cemented prostheses were most cost effective.
Conclusions Cemented prostheses were the least costly type for total hip replacement, but for most patient groups hybrid prostheses were the most cost effective. Cementless prostheses did not provide sufficient improvement in health outcomes to justify their additional costs.

\section{Introduction}

Total hip replacement is one of the most common surgical procedures. In 2010 the global market for hip prostheses was estimated at $\$ 4.7 b$ ( $£ 3.0 b ; € 3.5 b){ }^{1}$ A large number of different prosthesis designs have been developed and introduced on the market. For example, in England and Wales in 2010 at least 123 different brands of acetabular cups and 146 brands of femoral stems were used. ${ }^{2}$ These prosthesis brands are often grouped into cemented, cementless, and hybrid prostheses. Hybrid prostheses consist of cemented stems and cementless cups.

Cementless prostheses, although the most expensive, have become the most common type of prosthesis used for total hip replacement in England, Wales, Italy, Australia, Canada, and the United States, with hybrid prostheses growing in popularity. ${ }^{2-6}$ The increasing use of cementless components has contributed to a doubling of prosthesis costs between 1996 and $2006 .^{7}$ Although a recent study analysing data from the National Joint Registry for England and Wales, the largest orthopaedic registry in the world, suggested that cementless prostheses might be associated with lower mortality than cemented prostheses, ${ }^{8}$ evidence to assess whether the increased costs of cementless components are justified by improved health outcomes is lacking. 
Randomised controlled trials have compared revision rates across prosthesis types, but with insufficient sample sizes or durations of follow-up to produce conclusive results. ${ }^{9}$ The largest observational study found that seven year revision rates were lower for cemented (3.0\%) than for hybrid (3.8\%) or cementless prostheses $(4.6 \%){ }^{2}$

Previous economic evaluations comparing prosthesis types suggested that cementless prostheses are relatively cost effective. ${ }^{10-12}$ These studies did not, however, consider differences between prosthesis types in preoperative case mix $^{11}$ or postoperative quality of life. ${ }^{10} 12$

We evaluated the relative cost effectiveness of cemented, cementless, and hybrid prostheses for elective total hip replacement surgery. The study took a health service perspective and presents lifetime cost effectiveness results for men and women aged 60,70 , and 80 . To overcome the deficiencies of earlier economic analyses, we used data on case mix and quality of life from a national programme that collects patient reported outcome measures in patients undergoing an elective total hip replacement in the English National Health Service, ${ }^{13}$ linked to records of the national joint registry.

\section{Methods \\ Model overview}

We used a Markov model with a cycle length of one year to simulate transitions between health states over the patients' lifetime. ${ }^{14}$ For each prosthesis type we estimated costs and outcomes for a hypothetical cohort of patients who enter the model at the time of the primary total hip replacement (fig $1 \Downarrow$ ). After the primary replacement, patients face a possibility of immediate postoperative mortality and annual probabilities of revision of the total hip replacement (one stage or two stage) and all cause mortality. If a prosthesis fails, the model assumes patients will have their hip prosthesis revised.

We summed the time in each health state over the annual cycles, weighted for quality of life, to estimate life expectancy in terms of quality adjusted life years (QALYs). Lifetime costs were calculated by adding costs that were related to the primary hip replacements to the costs of one stage and two stage revisions. The main model assumptions, summarised in the box, were taken from a previous cost effectiveness analysis. ${ }^{15}$ In sensitivity analyses we tested whether our results are robust to alternative assumptions. We describe data sources and statistical analyses for the main model parameters.

\section{Data sources}

\section{Overview}

For the cost effectiveness analysis model we estimated several parameters from individual patient data: quality of life (after primary total hip replacement, revised total hip replacement), length of stay after primary total hip replacement, rates of revision and re-revision, and mortality. The three data sources used were the national patient reported outcome measures programme, the National Joint Registry for England and Wales, and hospital episode statistics. Hospital episode statistics is the administrative database of all NHS funded hospital admissions in England. ${ }^{16}$

We used data on patient reported outcome measures for the estimation of quality of life after each type of total hip replacement. As we required data from the joint registry to categorise prosthesis type, we excluded those records on patient reported outcome measures without a record linked to the registry. All patient reported outcome measures entries with a joint registry record were also linked to hospital episode statistics (patient reported outcome measures, national joint registry, and hospital episode statistics data). From these linked records we estimated the length of stay after total hip replacement. We considered primary and revision operations separately.

To provide the most accurate estimates of revision rates within the first five years by prosthesis type, we used the joint registry data but excluded patients without a linked record to hospital episode statistics (joint registry and hospital episode statistics data). From unlinked data in hospital episode statistics we estimated the rates of revision after five years, re-revision, and mortality (hospital episode statistics only). See the supplementary file for further information on data sources and exclusion criteria required to estimate each parameter.

\section{Quality of life, preoperative characteristics, and prosthesis type}

We obtained data on quality of life from patients who had an elective total hip replacement between July 2008 and December 2010 in the English NHS. ${ }^{13}$ The database of patient reported outcome measures provided data on comorbidities, preoperative and postoperative symptoms and disability (Oxford hip score), and quality of life (EQ-5D-3L). The Oxford hip score is a disease specific instrument with 12 questions and responses expressed on a four point scale to produce an overall score ranging from 0 (worst health status) to 48 (best health status). ${ }^{17}$ The EQ-5D-3L is a generic instrument with five dimensions of health (mobility, self care, usual activities, pain and discomfort, anxiety and depression) and three levels (no problems, some problems, severe problems). We combined the EQ-5D-3L profiles with health state preference values from the UK general population, to give EQ-5D-3L utility index scores on a scale anchored at 0 (death) and 1 (perfect health). ${ }^{18}$ We accessed preoperative records on patient reported outcome measures for 73666 adults after excluding those aged under 55 or over 84 , and we subsequently included 39734 patients with a linked joint registry record in the estimation of quality of life after primary total hip replacement. The joint registry provided data on prosthesis type, diagnosis (osteoarthritis or other), body mass index, and American Society of Anesthesiologists (ASA) grade for physical status. ${ }^{19}$ Hospital episode statistics provided data on socioeconomic deprivation, derived from the patient's postcode, as the index of multiple deprivation. ${ }^{20} \mathrm{We}$ used five socioeconomic groups based on fifths of the national ranking of areas with an average population of 1500 people.

For the estimation of quality of life after primary total hip replacement, we excluded those who did not have a diagnosis of osteoarthritis; who received prostheses with a cementless stem and a cemented acetabular cup, or a hip resurfacing prosthesis; who had bone grafts; who had image guided or minimally invasive surgery; who had a bilateral procedure; or whose joint replacement was privately funded. We also excluded patients who had died, because death is captured separately in the Markov model. The resulting sample of 30203 patients was used to estimate quality of life six months after each type of total hip replacement. The model applies these quality of life estimates to patients in the primary total hip replacement health state in the initial cycle and each subsequent cycle.

For the health state during the year in which patients had a revision total hip replacement because of a prosthesis failure, we took quality of life from data on preoperative patient reported outcome measures for 2105 patients with linked data between patient reported outcome measures, joint registry, and hospital 


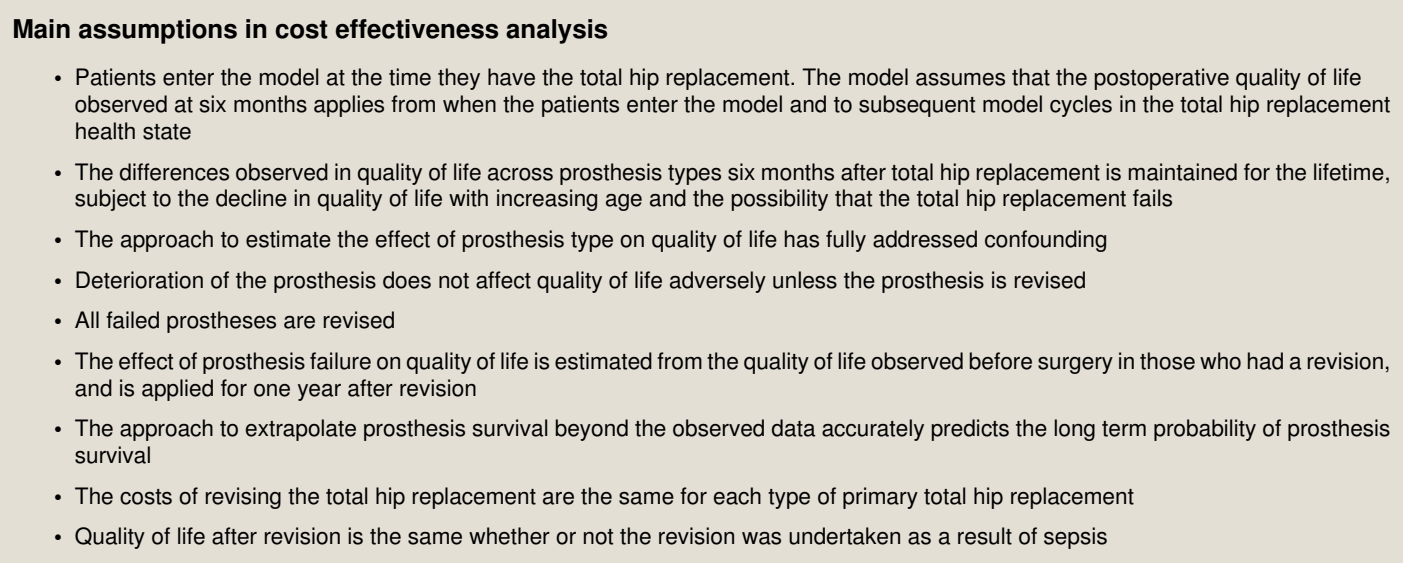

episode statistics who had a revision. For subsequent years after revision, when patients were assumed to be in the revised total hip replacement health state, we took quality of life from the linked data collected for 1283 patients six months after revision surgery.

\section{Rates of revision and re-revision}

To estimate revision rates we exploited access to all of the joint registry records on total hip replacement since inception in 2003 until December 2009 and hospital episode statistics data from April 1997 until December 2009. Where possible we linked the patient records in the joint registry to the corresponding record in hospital episode statistics to create a linked dataset for these two repositories. From these linked records we estimated the annual probabilities of revision in the first five years after total hip replacement. From the joint registry we accessed 289785 records of patients who had a primary total hip replacement between 1 April 2003 and 31 December 2009, of which 216 693 could be linked to hospital episode statistics. Records for 144661 patients were available for analysis after applying the same exclusion criteria applied to the dataset of patient reported outcome measures, joint registry, and hospital episode statistics linked records. We considered a patient to have had a revision if we found a record of revision surgery in either the joint registry or hospital episode statistics. Overall, 2023 revisions were identified: 866 for cemented prostheses (1.2\%), 817 for cementless prostheses $(1.8 \%)$, and 340 for hybrid prostheses (1.4\%). Data capture in the joint registry was poor before 2005, hence revision rates after five years were based on data from hospital episode statistics only. After applying the previously mentioned exclusion criteria for age and diagnosis we identified 201655 eligible patients who had a primary total hip replacement carried out between 1 March 1997 and 24 December 2004. We estimated the rates of re-revision from the records of 54134 patients with a revision recorded in hospital episode statistics between April 1997 and December 2009.

\section{Mortality}

Mortality (surgical and all cause) in the year after total hip replacement was estimated from hospital episode statistics data. Subsequent annual mortality (all cause) according to age and sex was taken from general population data, after using information from hospital episode statistics data to adjust for the "healthy patient" effect, given that patients who undergo a total hip replacement are likely to have a slightly lower mortality than that observed in the general population. ${ }^{21} \mathrm{We}$ did not find any difference in mortality across prosthesis types after adjusting for potential confounders (age, sex, ASA grade, body mass index, articulation type, funding source, and date of surgery). The hazard rate for mortality after total hip replacement using cemented versus cementless prostheses was $1.01(\mathrm{P}=0.75)$, and so we applied the same probabilities of death across all prosthesis types.

\section{Costs}

The unit cost of each prosthesis type was taken from the prices paid by a typical NHS provider for the most popular implant systems, including all components and instrumentation. These prices were lower than list prices. We calculated unit costs for each prosthesis type by weighting the price for each brand within the different types according to each brand's relative frequency, assuming a standard metal-on-polythene articulation: $£ 739$ for a cemented prosthesis, $£ 1697$ for a cementless prosthesis, and $£ 1285$ for a hybrid prosthesis.

Costs of the operation theatre and hospital stay were based on a national study, ${ }^{22}$ modified by using the length of stay according to prosthesis type that we observed in the patient reported outcome measures, joint registry, and hospital episode statistics linked records ( $\mathrm{n}=30203)$, and assuming a cost per hospital day of $£ 225 .{ }^{23}$ Unit costs of revisions are generally higher than for the initial surgery ${ }^{24}$; here we multiplied the average total cost of a primary total hip replacement by a factor that differed according to the reason for revision (3.6 for two stage revisions and 1.4 for one stage revisions). ${ }^{25}$ All unit costs were reported in British pounds ( $£ 1.00$; $\$ 1.60 ; € 1.20)$ according to $2010-11$ prices.

\section{Statistical analysis to provide input parameters for cost effectiveness model Quality of life after primary total hip replacement
and revision}

We estimated quality of life following primary total hip replacement according to prosthesis type after adjusting for observed differences in preoperative characteristics between the comparison groups. For the adjustment we used both Genetic Matching (GenMatch) and regression. GenMatch is a multivariate matching method that aims to make the distribution of baseline characteristics as similar as possible, ${ }^{26}$ and it has previously been used in evaluating relative effectiveness and cost effectiveness. ${ }^{27-29}$ GenMatch selects matched pairs using a generalised Mahalanobis distance metric, which weights each baseline covariate included in the matching. The weights define alternative distance metrics, which differ in the relative importance given to matching each covariate. The automated search algorithm selects those weights (and hence the corresponding distance metric) that give the best covariate 
balance in the matched samples. The balance statistics are chosen, a priori, from recommended measures such as $t$ statistics from paired $t$ tests, D statistics from Kolmogorov-Smirnov tests, and weighted standardised differences. The search algorithm optimises the balance between covariates within the constraints of the data. Compared with propensity score matching, GenMatch has been shown to reduce imbalance between covariates and bias from confounding. ${ }^{26} 2730$

For patients receiving each prosthesis type, we used GenMatch to find the best match from each of the other two prosthesis groups. Patients were matched on age, sex, number of comorbidities, body mass index, disability, ASA grade, index of multiple deprivation, preoperative EQ-5D-3L and Oxford hip scores, surgeon experience (senior surgeon or not), and hospital type (treatment centre or not). All matching was performed as nearest neighbour matching one to one with replacement. We used linear regression to adjust for any remaining imbalance and to predict postoperative quality of life by prosthesis type for each subgroup (for men and women aged 60,70 , and 80 ). The model included the above covariates but with ASA grade categorised as grades 1, 2, and 3-5, and body mass index (weight $(\mathrm{kg}) /\left(\right.$ height $\left.(\mathrm{m})^{2}\right)$ ) as less than $30,30-35$, and greater than 35 . The model also included quadratic terms for age, and quadratic and cubic terms for preoperative EQ-5D-3L scores. We defined reference groups by the average preoperative quality of life for each subgroup, a body mass index lower than 30, and an ASA grade of 2 (mild systemic disease).

We used regression to predict the mean quality of life in the year of revision surgery as a function of age, sex, and type of revision (one stage or two stage). Regression was also used to predict the mean quality of life after revision as a function of age and sex.

To estimate the effect of aging on quality of life after primary total hip replacement we applied an ordinary least squares regression model using data from the medical expenditure panel survey. ${ }^{31} \mathrm{We}$ found that EQ-5D-3L scores declined by on average 0.0033 for an increase in age of one year. Hence, quality of life after primary total hip replacement was reduced by 0.0033 for each year that patients survived beyond the first. As quality of life after revision was already parameterised as a function of age, we did not undertake further adjustment for aging.

Of the 30203 patients included in the quality of life analysis, data were missing on body mass index for $36 \%$ and on postoperative patient reported outcome measures for $33 \%$. All other data items were complete for over $90 \%$ of the sample. We applied multiple imputation using chained equations to preoperative and postoperative data to impute missing responses. ${ }^{32}$ Five imputations were undertaken and we used Rubin's rules to combine the results across these imputations.

\section{Rates of revision and re-revision}

The annual revision rates within the first five years were predicted from linked joint registry and hospital episode statistics data for each prosthesis type, adjusted for case mix (age, sex, ASA grade, and body mass index). We used a piecewise constant survival regression model, which assumes constant hazards within one year periods to capture the underlying variation in revision rates over time without imposing a prespecified relation. Revisions predicted by the survival model were further classified as one stage or two stage. We predicted the probability of whether or not the revision of a prosthesis was one stage according to joint registry classifications, with logistic regression.
Revision rates for cemented and cementless prostheses beyond five years after the total hip replacement were predicted by fitting a survival model with a Weibull hazard function to patients in hospital episode statistics with five to 12 years of follow-up data, adjusting for age and sex. Comparison of records in hospital episode statistics alone with the joint registry and hospital episode statistics linked record indicated a slightly lower capture of revisions in the absence of joint registry linkage. Consequently, we applied a weighting factor of 1.15 to revision probabilities estimated from hospital episode statistics. The hospital episode statistics data do not identify hybrid prostheses as a distinct category before 2006, so after five years we assumed the revision rates for hybrid prostheses were the same as for cemented or cementless prostheses, whichever was the highest.

Re-revision rates were estimated with a piecewise constant survival regression model, which allowed for different revision rates in the first year versus subsequent years. All re-revisions were assumed to be one stage revisions.

Regression analysis was undertaken in Stata version 12. Markov modelling was undertaken in Microsoft Excel.

\section{Cost effectiveness analysis}

The cost effectiveness model estimated lifetime revision rates, costs related to total hip replacement, and QALYs for men and women aged 60,70 , and 80 , according to the reference group defined in the regression models (the average preoperative quality of life for each subgroup, body mass index $<30$, and ASA grade 2). We report the incremental costs per QALY and the probability that each prosthesis type is the most cost effective. By undertaking a probabilistic analysis we recognised sampling uncertainty around the model parameters. Model results are reported after averaging across 1000 simulations in which each model parameter was sampled from the appropriate probability distribution.

In each simulation the net monetary benefit was calculated for each prosthesis type by multiplying the lifetime QALYs by society's willingness to pay for a QALY gain and subtracting from this the total lifetime cost. The willingness to pay thresholds ranged from $£ 0$ to $£ 50000$. We calculated cost effectiveness acceptability curves to report the proportion of simulations that each prosthesis type is the most cost effective - that is, had the highest net monetary benefit - as a function of the threshold willingness to pay. Future costs and outcomes should be valued less than costs and outcomes immediately after the total hip replacement to reflect societal time preferences. We applied a recommended annual discount rate of $3.5 \%$ to both costs and outcomes.

\section{Sensitivity analyses}

We tested whether our results were robust to alternative assumptions. Firstly, the base case model assumes that in the absence of a prosthesis failure, any differences in quality of life between prosthesis types are maintained over the patient's lifetime. Here we assumed instead that any residual differences in quality of life between the prosthesis types are maintained for two years after the total hip replacement. Secondly, to consider an alternative way of addressing potential confounding, we estimated postoperative quality of life with ordinary least squares regression but without using GenMatch. Thirdly, we predicted revision rates beyond five years using a Gompertz rather than a Weibull hazard function. Fourthly, we created a "failed hip" state, whereas in the base case analysis we assumed that all patients with a prosthetic failure had a revision. Patients 
were assumed to move to this failed hip state if their prosthesis failed and their risk of operative mortality was predicted to exceed $10 \%$. Quality of life for this failed hip state was assumed to be that for patients awaiting revision surgery. Fifthly, in the light of recent concerns over the use of metal-on-metal prosthese ${ }^{33}$ we undertook a sensitivity analysis in which quality of life and revision rates after total hip replacement were estimated after exclusion of patients with these prostheses.

\section{Results}

\section{Patient characteristics before total hip replacement}

Of the 30203 patients included in the analysis of quality of life after total hip replacement, those who received a cemented prosthesis were on average older, more likely to be women, and to have more comorbidities and a higher ASA grade than those who received another prosthesis type (table $1 \Downarrow$ ). The mean preoperative Oxford hip score and EQ-5D-3L index score were slightly higher in the group that received cementless or hybrid prostheses.

\section{Initial outcomes and costs after primary total hip replacement}

After matching, the preoperative characteristics were well balanced across the comparison groups. In 70 year old men and women, the mean quality of life six months after the primary total hip replacement was predicted to be lowest with cemented prostheses and highest with hybrid prostheses (table $2 \Downarrow$ ). The differences in postoperative outcome across prosthesis types were reflected in both quality of life (EQ-5D-3L index score) and hip symptoms and function (Oxford hip score). The same pattern was observed in 60 year old men and women and 80 year old men.

Results from the sensitivity analysis in which ordinary least squares regression was undertaken without GenMatch gave similar results to the base case. Hybrid prostheses were predicted to provide the highest postoperative quality of life for all subgroups except women aged 80.

In men and women aged 70 and 80 revision rates at five and 10 years were lowest with cemented prostheses and highest with cementless prostheses. Only in 60 year old men were cementless prostheses competitive in terms of 10 year revision rates. In all subgroups, initial costs were lowest with cemented prostheses and highest with cementless prostheses.

\section{Cost effectiveness analysis}

In men and women aged 70 the average lifetime costs related to total hip replacement were lowest with cemented prostheses and highest with cementless prostheses (table $3 \Downarrow$ ). In this age group, patients with cementless or hybrid prostheses had slightly higher expected QALYs. Hybrid prostheses were reported to produce higher expected QALYs at lower overall cost than cementless prostheses. The incremental cost effectiveness ratio for a hybrid prosthesis compared with a cemented prosthesis was about $£ 2100$ per QALY for men and $£ 2500$ for women.

Similarly, the cost effectiveness acceptability curves in figure $2 \Downarrow$ and the values in table $4 \Downarrow$ show that hybrid prostheses were most likely to be cost effective for patients aged 70-that is, hybrid prostheses were associated with the highest net monetary benefit in most of the 1000 simulations in the probabilistic model. If the societal willingness to pay per QALY was $£ 10$ 000 or higher, the probabilities that hybrid prostheses were the most cost effective prosthesis type were about $75 \%$ for men and
$70 \%$ for women. The corresponding probabilities for cementless prostheses were about $25 \%$ for men and $30 \%$ for women, and around $1 \%$ for cemented prostheses.

For men aged 60 or 80 and for women aged 60, hybrid prostheses gave the highest expected net benefit and had the highest probability of being the most cost effective prosthesis type (fig 2 and table 4 ). For women aged 80, cemented prostheses were most cost effective.

\section{Sensitivity analyses}

The base case model was generally robust, in that the pattern of results remained similar with alternative assumptions, even if metal-on-metal prostheses were excluded (table $5 \Downarrow$ ). However, when the difference in quality of life between prosthesis types was assumed to last only two years, the pattern changed, but hybrid prostheses remained likely to be the most cost effective option for men and women aged 70 .

\section{Discussion}

In patients aged 70, which is about the mean age of those undergoing a total hip replacement for osteoarthritis, the incremental costs per quality adjusted life year (QALY) for hybrid prostheses compared with cemented prostheses was about $£ 2100$ for men and $£ 2500$ for women. In all subgroups, apart from women aged 80 , hybrid prostheses resulted in higher mean postoperative quality of life than cementless or cemented prostheses, and so have higher lifetime QALYs. At realistic levels of willingness to pay for a QALY gain, the probability that hybrid prostheses are the most cost effective option is around $75 \%$ for patients aged 70 . In patients aged 60 and 80 , the cost effectiveness results were less clear cut, but for 70 year olds the finding that the hybrid type of prosthesis was the most cost effective alternative was robust to alternative assumptions.

\section{Comparison with other studies}

The results of our cost effectiveness analysis differ from those of three previous economic evaluations, which suggested that cementless prostheses were cost effective compared with cemented ${ }^{11}{ }^{12}$ or hybrid prostheses. ${ }^{10}$ However, all those studies had serious methodological shortcomings. Only one study measured postoperative quality of life according to prosthesis type, ${ }^{11}$ but it was based on a small sample of patients $(n=363)$, did not include hybrid prostheses, was undertaken about two decades ago, and did not adjust for differences in key case mix factors such as preoperative quality of life and age. A second study did not seem to adjust for age when comparing revision rates across prostheses types, despite age differences of 4.5 years. ${ }^{10}$ The third study presented results that were inconsistent with their model parameters. ${ }^{12}$

\section{Strengths and limitations of this study}

Our cost effectiveness study had several strengths. Firstly, we estimated revision rates and postoperative quality of life by prosthesis type from large national studies. These sources provided detailed information about the patients' preoperative condition. Although the estimation of postoperative quality of life and five year revision rates required national joint registry linked data, our sample was representative of the target population of interest. Secondly, we used GenMatch, a sophisticated multivariate matching approach, combined with regression, to improve the case mix adjustment when estimating postoperative quality of life, a key driver of the cost effectiveness results. Thirdly, in taking a recommended decision 
analytical approach, ${ }^{15}$ we refined the structure of a previous model by allowing prosthesis failure to occur in the first year after the primary hip replacement or after revision surgery. We also distinguished between one and two stage revisions. Fourthly, our key model estimates represent current practice. The estimates of the revision rates, quality of life, and mortality were derived from patients who have undergone a hip replacement since 1997. This is potentially important as over the last two decades revision rates have decreased considerably. ${ }^{34}$ Fifthly, our cost estimates are based on the actual prices paid by mid-sized centres in the English NHS, as well as on the length of hospital stay observed for the different prosthesis types. Finally, we undertook a full probabilistic sensitivity analysis to ensure that the sampling uncertainties in the model parameters were fully represented in the results. We then tested the results' sensitivity to key assumptions.

A crucial step in the analysis was the estimation of lifetime outcomes. Lifetime revision rates were derived from the National Joint Registry of England and Wales and administrative data from all relevant hospital admissions to the English NHS with a maximum follow-up of 13 years, which provided a stronger basis for extrapolation than previous studies. ${ }^{10-12} \mathrm{~A}$ potential concern is the assumption that differences in quality of life observed at six months after the joint replacement remain unchanged for the rest of a patient's life, after allowing for increasing age and any differential revision rates between prosthesis types. The sensitivity analysis demonstrated that if the residual differences in postoperative quality of life were assumed to last for only two years, then the probability that cemented prostheses are cost effective increased. However, there is evidence that the quality of life improvements after total hip replacement persists for at least five years. ${ }^{35}$

We found that quality adjusted life expectancy was not only highest for hybrid prostheses but that this prosthesis type also had the highest lifetime revision rate. This shows that postoperative quality of life is an important driver of our results. It is therefore reassuring that the results from using the quality of life measure (EQ-5D-3L) are consistent with those of a disease specific score (Oxford hip score) that measured severity of symptoms and function. Unlike a previous study, our analysis of linked data from the national joint registry and hospital episode statistics with adjustment for patients and prosthesis characteristics, showed no difference in mortality across prosthesis types. ${ }^{8}$

This study has some limitations. Firstly, while we used the best available information on postoperative quality of life, and a sophisticated matching method to allow for all well recognised preoperative case mix factors, the possibility of bias from residual confounding remains. Secondly, previous work has highlighted that long term predictions from parametric survival models can be inaccurate. ${ }^{36}$ While this remains a concern, the sensitivity analysis found that the results were robust to the choice of extrapolation approach. Thirdly, this cost effectiveness analysis compared alternative prosthesis types; it did not consider that revision rates within a prosthesis type may differ by brand. ${ }^{2}$ Further research following the approach taken here will explore the lifetime cost effectiveness of alternative brands. Similarly, future studies will assess whether the additional costs of newer, more costly, joint bearing surfaces commonly used with cementless prostheses, are justified by improved revision rates or quality of life.

\section{Conclusions}

This study questions the increased use of cementless prostheses for total hip replacement. Cemented prostheses are the cheapest option, but hybrid prostheses lead to greater gains in mean postoperative quality of life and are the most cost effective alternative for most patients. Cementless prostheses do not improve health outcomes sufficiently to justify their higher costs.

We thank the patients and staff of the hospitals in England and Wales who contributed data to the National Joint Registry for England and Wales; the Healthcare Quality Improvement Partnership, the national joint registry steering committee, and staff at the national joint registry centre for facilitating this work. The authors have conformed to the national joint registry's standard protocol for data access and publication. The views expressed represent those of the authors and do not necessarily reflect those of the national joint register steering committee, the Health Quality Improvement Partnership, the NHS Information Centre, or the Department of Health who do not vouch for how the information is presented. Hospital episode statistics data have been re-used with the permission of The Health and Social Care Information Centre. The hospital episode statistics data linked to national joint registry data were extracted by Northgate Information Solutions (UK) in mid-March 2010. We are grateful to Philip Lewis at NHS SupplyChain for information on the cost of prostheses.

Contributors: NB and JHvdM conceived the study. JHvdM and RG designed the study. MP and JSS undertook the analysis. MP, RG, and JHvdM wrote the manuscript. NB, JSS, and PG commented on and revised drafts. JvdM is guarantor.

Funding: This work was supported by the Department of Health (Commissioning Development).

Competing interests: All authors have completed the ICMJE uniform disclosure form at http://www.icmje.org/coi_disclosure.pdf (available on request from the corresponding author) and declare: no support from any organisation for the submitted work; no financial relationships with any organisations that might have an interest in the submitted work in the previous three years, no other relationships or activities that could appear to have influenced the submitted work.

Ethical approval: The study is exempt UK National Research Ethics Service approval as it involved analysis of existing datasets of anonymised data for service evaluation. Approvals for the use of hospital episode statistics data were obtained as part of the standard hospitals episode statistics approval process.

Data sharing: No additional data available.

1 Hip replacement implants—global pipeline analysis, competitive landscape and market forecasts to 2017. MarketResearch.com, 2011.

2 National Joint Registry for England and Wales. National joint registry, 8th annual report. NJR, 2011.

3 Hip and knee replacements in Canada—Canadian Joint replacement Registry (CJRR) 2008-2009 annual report. Canadian Institute for Health Information, 2009.

4 Graves S, Davidson D, de Steiger R, Tomkins A, Ryan P, Griffith L, et al. Australian Orthopaedic Association National Joint Replacement Registry. Annual report. AOA, 2011.

5 Mendenhall S. Hip and knee implant review. Orthopedic News Network 2004;14:1-16.

6 Stea S, Bordini B, De Clerico M, Petropulacos K, Toni A. First hip arthroplasty register in Italy: 55,000 cases and 7 year follow-up. Int Orthop 2009;33:339-46.

Hip and knee implant list prices rise 6.3 percent. Orthopedic Network News 2008;19:1-9.

8 McMinn DJW, Snell KIE, Daniel J, Treacy RBC, Pynsent PB, Riley RD. Mortality and implant revision rates of hip arthroplasty in patients with osteoarthritis: registry based cohort study. 2012;344:e3319.

9 Morshed S, Bozic KJ, Ries MD, Malchau H, Colford Jr JM. Comparison of cemented and uncemented fixation in total hip replacement: a meta-analysis. Acta Orthop 2007;78:315-26.

10 Di Tanna GL, Ferro S, Cipriani F, Bordini B, Stea S, Toni A, et al. Modeling the cost-effectiveness for cement-less and hybrid prosthesis in total hip replacement in Emilia Romagna, Italy. J Surg Res 2010;169:227-33.

11 Givon U, Ginsberg GM, Horoszowski H, Shemer J. Cost-utility analysis of total hip arthroplasties: technology assessment of surgical procedures by mailed questionnaires. Int J Technol Assess Health Care 1998;14:735-42.

12 Marinelli M, Soccetti A, Panfoli N, Palma L. Cost-effectiveness of cemented versus cementless total hip arthroplasty. A Markov decision analysis based on implant cost. J Orthop Traumatol 2008;9:23-8. 


\section{What is already known on this topic}

Total hip replacement is highly cost effective compared with alternative medical management of osteoarthritis

Cemented prostheses are generally cheaper than hybrid or cementless prostheses, and there is some evidence that failure rates are lower in patients over 55

Previous economic analyses all have serious limitations

\section{What this study adds}

Lifetime costs were lowest with cemented prostheses and highest with cementless prostheses

On average, hybrid prostheses were associated with the highest postoperative health related quality of life, provided the highest lifetime QALYs, and were the most cost effective prosthesis type, except in women aged 80 or more

Cementless prostheses were not cost effective in patients with osteoarthritis

13 Provisional Monthly Patient Reported Outcome Measures (PROMs) in England: April 2009-April 2010: pre- and post-operative data: experimental statistics. Health and Social Care Information Centre, 2010.

14 Briggs A, Sculpher M. An introduction to Markov modelling for economic evaluation. Pharmacoeconomics 1998;13:397-409.

15 Briggs A, Sculpher M, Dawson J, Fitzpatrick R, Murray D, Malchau $H$. The use of probabilistic decision models in technology assessment: the case of total hip replacement. Applied Health Economics Health Policy 2004:3:79-89.

16 Hospital Episode Statistics for England. Inpatient statistics: NHS Information Centre; 2009

17 Dawson J, Fitzpatrick R, Carr A, Murray D. Questionnaire on the perceptions of patients about total hip replacement. J Bone Joint Surg Br 1996;78:185-90.

18 EuroQol—a new facility for the measurement of health-related quality of life. The EuroQol Group. Health Policy 1990;16:199-208.

19 ASA Physical Status Classification System. American Society of Anesthesiologists

20 Noble M, Mclennan D, Wilkinson K, Whitworth A, Exley S, Barnes H, et al. The English Indices of Deprivation 2007. Department of Communities and Local Government, 2007.

21 Lie SA, Engesaeter LB, Havelin LI, Gjessing HK, Vollset SE. Mortality after total hip replacement: 0-10-year follow-up of 39,543 patients in the Norwegian Arthroplasty Register. Acta Orthop Scand 2000;71:19-27.

22 Lemon MA, Hamilton PD, Field RE. Comparing total hip and knee replacement costs. $\mathrm{Br}$ $J$ Healthcare Manage 2008;14:108-12.

23 Length of stay-reducing length of stay. National Health Service, Institute for Innovation and Improvement, 2008.

24 Metz CM, Freiberg AA. An international comparative study of total hip arthroplasty cost and practice patterns. J Arthroplasty 1998;13:296-8.

25 Klouche S, Sariali E, Mamoudy P. Total hip arthroplasty revision due to infection: a cost analysis approach. Orthop Traumatol: Surg Res 2010:96:124-32.

26 Sekhon JS, Grieve R. A matching method for improving covariate balance in cost-effectiveness analyses. Health Econ 2011;21:695-714.

27 Grieve R, Sekhon JS, Hu T-W, Bloom JR. Evaluating health care programs by combining cost with quality of life measures: a case study comparing capitation and fee for service. Health Serv Res 2008;43:1204-22.

28 Kreif N, Grieve R, Radice R, Sadique Z, Ramsahai R, Sekhon JS. Methods for estimating subgroup effects in cost-effectiveness analyses that use observational data. Med Decis Making 2012 16/10/12. http://mdm.sagepub.com/content/early/2012/06/11/ $0272989 \times 12448929$.
29 Radice R, Ramsahai R, Grieve R, Kreif N, Sadique Z, Sekhon JS. Evaluating treatment effectiveness in patient subgroups: a comparison of propensity score methods with an automated matching approach. Int J Biostat 2012;8:article 25.

30 Diamond A, Sekhon JS. Genetic matching for estimating causal effects: a general multivariate matching method for achieving balance in observational studies. review of economics and statistics. 2012. www.mitpressjournals.org/doi/abs/10.1162/REST_a_ 00318.

31 Fryback D, Hanmer J, eds. Do large national surveys yield equivalent population norms for health related quality of life measures? Society for Medical Decision Making, 2005; San Fransisco, CA.

32 White IR, Royston P, Wood AM. Multiple imputation using chained equations: issues and guidance for practice. Stat Med 2011;30:377-99.

33 Smith AJ, Dieppe P, Vernon K, Porter M, Blom AW. Failure rates of stemmed metal-on-metal hip replacements: analysis of data from the National Joint Registry of England and Wales. Lancet 2012;379:1199-204.

34 Fevang B-TS, Lie SA, Havelin LI, Engesaeter LB, Furnes O. Improved results of primary total hip replacement. Results from the Norwegian Arthroplasty Register, 1987-2007. Acta Orthopaedica 2010;81:649-59.

$35 \mathrm{Ng} \mathrm{CY}$, Ballantyne JA, Brenkel IJ. Quality of life and functional outcome after primary total hip replacement. A five-year follow-up. J Bone Joint Surg Br 2007;89:868-73.

36 Davies C, Briggs A, Lorgelly P, Garellick G, Malchau H. The 'hazards' of extrapolating survival curves. Presented at Health Economics Study Group meeting, Sheffield, UK, 2009.

Accepted: 31 January 2013

\section{Cite this as: BMJ 2013;346:f1026}

This is an open-access article distributed under the terms of the Creative Commons Attribution Non-commercial License, which permits use, distribution, and reproduction in any medium, provided the original work is properly cited, the use is non commercial and is otherwise in compliance with the license. See: http://creativecommons.org/licenses/bync/2.0/ and http://creativecommons.org/licenses/by-nc/2.0/legalcode. 


\section{Tables}

Table 1| Preoperative characteristics of $\mathbf{3 0} 203$ patients with linked records from patient reported outcome measures, National Joint Registry for England and Wales, and hospital episode statistics after multiple imputation. Values are numbers (percentages) unless stated otherwise

\begin{tabular}{lcccc} 
& \multicolumn{3}{c}{ Prosthesis type } & \\
\cline { 2 - 4 } Characteristics & Cemented & Cementless & Hybrid & Data source \\
\hline No of patients & 11955 & 14697 & 3551 & National joint registry \\
\hline Mean (SD) age (years) & $72.4(6.7)$ & $67.8(7.2)$ & $70.4(7.2)$ & \\
\hline Women & $7760(64.9)$ & $8149(55.4)$ & $2201(62.0)$ & National joint registry \\
\hline Most deprived fifth ${ }^{*}$ & $2309(19.3)$ & $2856(19.4)$ & $874(24.6)$ & Hospital episode statistics \\
\hline 22 comorbidities $\dagger$ & $2309(25.0)$ & $3197(21.8)$ & $866(24.4)$ & Patient reported outcome measures \\
\hline ASA grade $\geq 3$ & $2102(17.6)$ & $1815(12.3)$ & $558(15.7)$ & National joint registry \\
\hline Mean (SD) body mass index & $28.7(5.37)$ & $29.2(5.42)$ & $28.8(5.56)$ & National joint registry \\
\hline Hip replacement at independent sector treatment centre & $1099(9.2)$ & $1566(10.7)$ & $173(4.9)$ & Hospital episode statistics \\
\hline Hip replacement by consultant & $9873(82.6)$ & $12649(86.1)$ & $2871(80.9)$ & National joint registry \\
\hline Mean (SD) preoperative Oxford hip score & $17.7(8.12)$ & $18.3(8.24)$ & $18.1(8.16)$ & Patient reported outcome measures \\
\hline Mean (SD) EQ-5D-3L indexł & $0.34(0.32)$ & $0.36(0.32)$ & $0.34(0.32)$ & Patient reported outcome measures \\
\hline
\end{tabular}

ASA=American Society of Anesthesiologists.

*Multiple index of deprivation.

†Excludes osteoarthritis.

$\ddagger 0$ represents death and 1 perfect health. 


\begin{tabular}{|c|c|c|c|c|c|c|c|c|c|}
\hline \multirow[b]{2}{*}{ Variables } & \multicolumn{3}{|c|}{ Age 60} & \multicolumn{3}{|c|}{ Age 70} & \multicolumn{3}{|c|}{ Age 80} \\
\hline & Cemented & Cementless & Hybrid & Cemented & Cementless & Hybrid & Cemented & Cementless & Hybrid \\
\hline \multicolumn{10}{|l|}{ Men: } \\
\hline EQ-5D-3L index & 0.797 & 0.807 & 0.810 & 0.819 & 0.836 & 0.848 & 0.797 & 0.804 & 0.824 \\
\hline Oxford hip score & 40.0 & 40.4 & 40.9 & 40.4 & 41.0 & 41.6 & 39.7 & 40.4 & 41.1 \\
\hline Initial cost $(£) \dagger$ & 5996 & 6811 & 6610 & 6096 & 6919 & 6711 & 6459 & 7227 & 6989 \\
\hline $\begin{array}{l}5 \text { year revision } \\
\text { rate‡ (\%) }\end{array}$ & 2.4 & 3.1 & 2.5 & 2.0 & 2.9 & 2.3 & 1.8 & 3.3 & 3.3 \\
\hline $\begin{array}{l}10 \text { year revision } \\
\text { rate§ }(\%)\end{array}$ & 6.7 & 6.7 & 6.9 & 4.8 & 5.2 & 5.1 & 3.3 & 4.5 & 4.7 \\
\hline \multicolumn{10}{|l|}{ Women: } \\
\hline EQ-5D-3L index & 0.785 & 0.787 & 0.800 & 0.781 & 0.799 & 0.805 & 0.754 & 0.749 & 0.751 \\
\hline Oxford hip score & 39.8 & 40.1 & 40.3 & 39.1 & 40.0 & 40.3 & 38.3 & 38.4 & 38.3 \\
\hline Initial cost $(£) \dagger$ & 6065 & 6882 & 6694 & 6193 & 7018 & 6822 & 6581 & 7351 & 7126 \\
\hline $\begin{array}{l}5 \text { year revision } \\
\text { rate‡ }(\%)\end{array}$ & 1.8 & 2.9 & 2.1 & 1.5 & 2.8 & 1.9 & 1.4 & 3.1 & 2.7 \\
\hline $\begin{array}{l}10 \text { year revision } \\
\text { rate§ }(\%)\end{array}$ & 5.1 & 5.6 & 5.3 & 3.6 & 4.5 & 4.0 & 2.5 & 4.0 & 3.8 \\
\hline
\end{tabular}

*After applying GenMatch and regression to allow for observed preoperative differences.

†Combined costs of prosthesis, operating theatre, and hospital stay.

$\ddagger$ After adjusting for case mix by applying piecewise constant hazards model to linked data from the National Joint Registry of England and Wales and hospital episode statistics.

§After adjusting for case mix by applying piecewise constant hazards model to linked data from the National Joint Registry of England and Wales and hospital episode statistics, (years 1-5) and by applying Weibull hazard function to data from hospital episode statistics (years 6-10). 
Table 3| Estimated lifetime revision rates, replacement related cost, and quality adjusted life years (QALYs) according to prosthesis type for men and women aged 60,70 , and 80

\begin{tabular}{|c|c|c|c|c|c|c|c|c|c|}
\hline \multirow[b]{2}{*}{ Sex and age } & \multicolumn{3}{|c|}{ Proportion revised (\%) } & \multicolumn{3}{|c|}{ Mean cost $(£)$} & \multicolumn{3}{|c|}{ Mean QALYs } \\
\hline & Cemented & Cementless & Hybrid & Cemented & Cementless & Hybrid & Cemented & Cementless & Hybrid \\
\hline \multicolumn{10}{|l|}{ Men: } \\
\hline 60 & 31.7 & 28.5 & 31.7 & 8167 & 8748 & 8726 & 11.06 & 11.21 & 11.23 \\
\hline 70 & 9.2 & 9.0 & 9.5 & 6912 & 7712 & 7516 & 8.46 & 8.63 & 8.75 \\
\hline 80 & 3.0 & 4.1 & 4.3 & 6819 & 7690 & 7481 & 5.22 & 5.25 & 5.38 \\
\hline \multicolumn{10}{|l|}{ Women: } \\
\hline 60 & 29.3 & 26.5 & 29.5 & 7864 & 8551 & 8487 & 11.69 & 11.73 & 11.90 \\
\hline 70 & 8.3 & 8.4 & 8.6 & 6837 & 7704 & 7486 & 8.99 & 9.18 & 9.25 \\
\hline 80 & 2.7 & 4.0 & 3.9 & 6853 & 7762 & 7521 & 5.82 & 5.75 & 5.78 \\
\hline
\end{tabular}

$£ 1.00(\$ 1.60 ; € 1.20)$ according to $2010-11$ prices. 
Table 4| Probability that a prosthesis type is cost effective at alternative willingness to pay thresholds for men and women aged 60,70 , and 80

\begin{tabular}{|c|c|c|c|c|c|c|c|c|c|}
\hline \multirow[b]{3}{*}{ Sex and age } & \multicolumn{9}{|c|}{ Willingness to pay thresholds } \\
\hline & \multicolumn{3}{|c|}{$£ 10000$} & \multicolumn{3}{|c|}{$£ 20000$} & \multicolumn{3}{|c|}{$£ 30000$} \\
\hline & Cemented & Cementless & Hybrid & Cemented & Cementless & Hybrid & Cemented & Cementless & Hybrid \\
\hline \multicolumn{10}{|l|}{ Men: } \\
\hline 60 & 0.16 & 0.35 & 0.49 & 0.13 & 0.37 & 0.50 & 0.12 & 0.38 & 0.50 \\
\hline 70 & 0.04 & 0.21 & 0.76 & 0.01 & 0.23 & 0.76 & 0.01 & 0.24 & 0.75 \\
\hline 80 & 0.13 & 0.07 & 0.80 & 0.04 & 0.11 & 0.85 & 0.03 & 0.12 & 0.86 \\
\hline \multicolumn{10}{|l|}{ Women: } \\
\hline 60 & 0.27 & 0.11 & 0.62 & 0.22 & 0.13 & 0.65 & 0.21 & 0.14 & 0.65 \\
\hline 70 & 0.03 & 0.23 & 0.74 & 0.01 & 0.25 & 0.74 & 0.01 & 0.26 & 0.73 \\
\hline 80 & 0.85 & 0.03 & 0.13 & 0.72 & 0.08 & 0.20 & 0.67 & 0.10 & 0.23 \\
\hline
\end{tabular}

$£ 1.00(\$ 1.60 ; € 1.20)$ according to $2010-11$ prices. 
Table 5 Sensitivity analysis. Probability that each prosthesis type is cost effective under alternative assumptions at a willingness to pay threshold of $£ 20000$ per quality adjusted life year (QALY) for men and women aged 60,70 , and 80

\begin{tabular}{llll} 
& \multicolumn{3}{c}{ Prosthesis type } \\
\cline { 2 - 4 } Analyses by sex and age & Cemented & Cementless & Hybrid \\
QoL analysis using linear regression without matching & \\
\hline Men: & & \\
\hline 60 & 0.13 & 0.06 & 0.81 \\
\hline 70 & 0.00 & 0.09 & 0.90 \\
\hline 80 & 0.36 & 0.22 & 0.42 \\
\hline Women: & & & \\
\hline 60 & 0.24 & 0.08 & 0.68 \\
\hline 70 & 0.01 & 0.07 & 0.93 \\
\hline 80 & 0.58 & 0.11 & 0.31 \\
\hline
\end{tabular}

\begin{tabular}{llll}
\hline \multicolumn{4}{l}{ Differences in QoL by prosthesis type maintained for 2 years } \\
\hline Men: & & \\
\hline 60 & 0.26 & 0.44 & 0.30 \\
\hline 70 & 0.23 & 0.09 & 0.69 \\
\hline 80 & 0.58 & 0.01 & 0.41 \\
\hline Women: & & & \\
\hline 60 & 0.45 & 0.16 & 0.39 \\
\hline 70 & 0.44 & 0.03 & 0.54 \\
\hline 80 & 0.99 & 0.00 & 0.01 \\
\hline
\end{tabular}

\section{"Failed hip" state for patients with high operative mortality} Men:

\begin{tabular}{llll}
\hline 60 & 0.12 & 0.37 & 0.52 \\
\hline 70 & 0.01 & 0.22 & 0.77 \\
\hline 80 & 0.07 & 0.11 & 0.83 \\
\hline Women: & & & \\
\hline 60 & 0.23 & 0.14 & 0.63 \\
\hline 70 & 0.01 & 0.28 & 0.72 \\
\hline 80 & 0.69 & 0.08 & 0.23 \\
\hline
\end{tabular}

Extrapolating revision rates using Gompertz hazard

Men:

\begin{tabular}{llll}
\hline 60 & 0.12 & 0.40 & 0.49 \\
\hline 70 & 0.01 & 0.24 & 0.75 \\
\hline 80 & 0.06 & 0.13 & 0.81 \\
\hline Women: & & & \\
\hline 60 & 0.21 & 0.16 & 0.63 \\
\hline 70 & 0.01 & 0.26 & 0.73 \\
\hline 80 & 0.71 & 0.08 & 0.21 \\
\hline
\end{tabular}

Exclusion of patients with metal-on-metal prostheses

Men:

\begin{tabular}{llll}
\hline 60 & 0.11 & 0.40 & 0.48 \\
\hline 70 & 0.01 & 0.19 & 0.80 \\
\hline 80 & 0.08 & 0.00 & 0.92 \\
\hline Women: & & & \\
\hline 60 & 0.26 & 0.12 & 0.62 \\
\hline 70 & 0.01 & 0.26 & 0.73 \\
\hline 80 & 0.69 & 0.07 & 0.24 \\
\hline
\end{tabular}

QoL=quality of life 
Table 5 (continued)

\begin{tabular}{l} 
Analyses by sex and age \\
\cline { 2 - 3 } Cemented Cementless Hybrid \\
$£ 1.00(\$ 1.60 ; € 1.20)$ according to $2010-11$ prices. \\
\hline
\end{tabular}




\section{Figures}

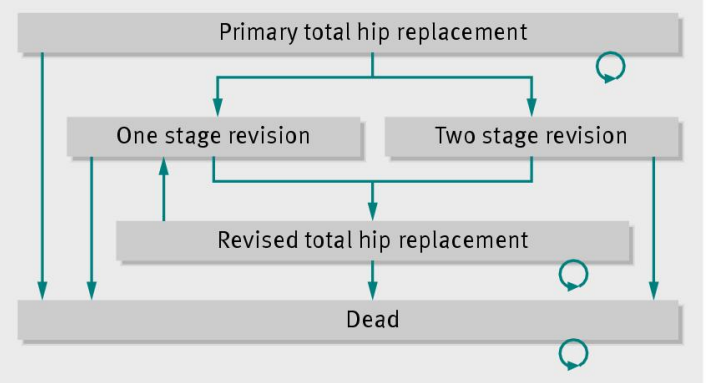

Fig 1 Markov model for cost effectiveness analysis of alternative prostheses types for primary total hip replacement
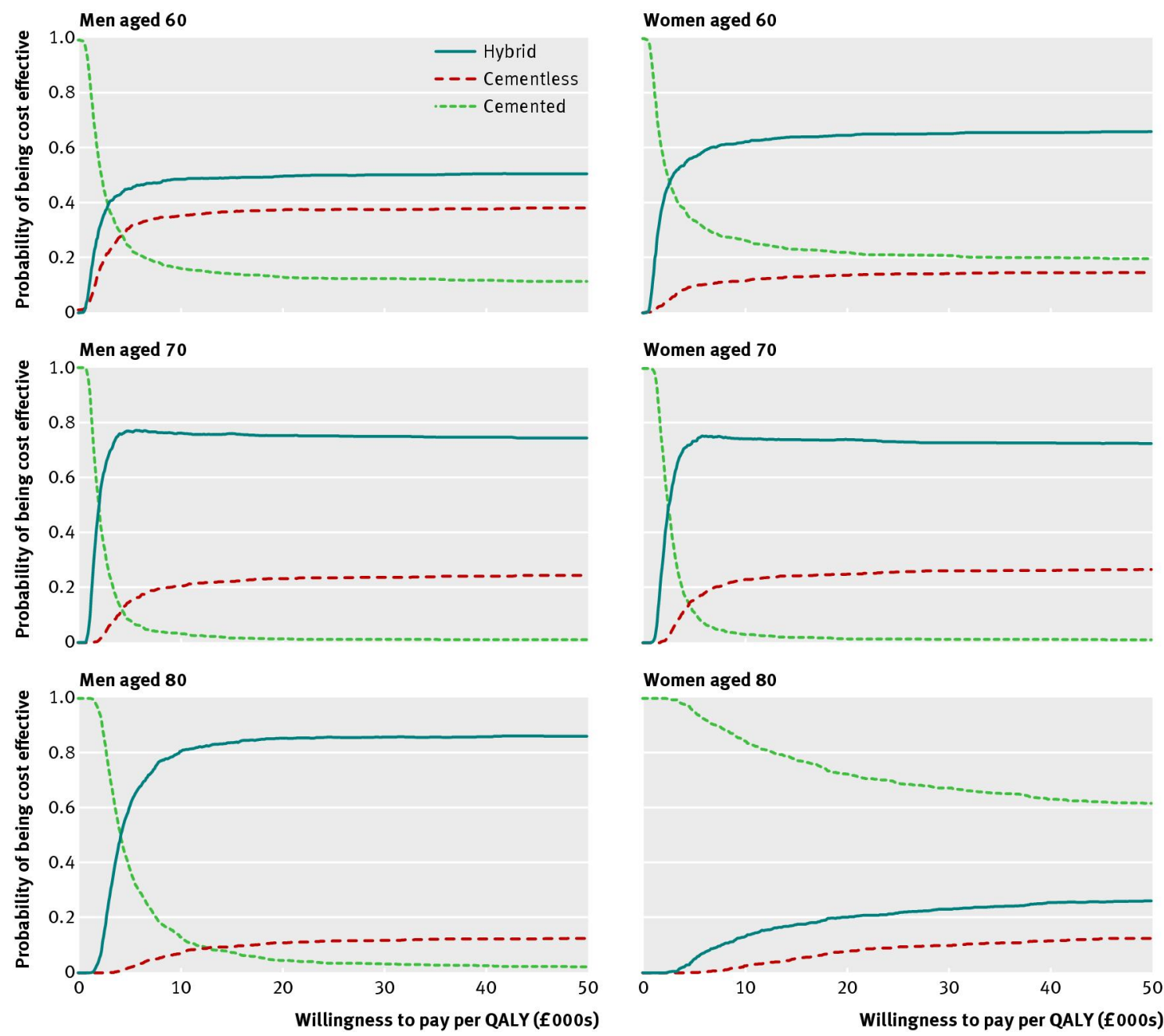

Fig 2 Cost effectiveness acceptability curves for cemented, cementless, and hybrid prostheses for total hip replacement by subgroup plotted for alternative threshold willingness to pay for a quality adjusted life year (QALY) gained 